\title{
Travelers with thyroid disorders
}

\author{
Wiktoria Stańska ${ }^{1}$, Krzysztof Korzeniewski' $^{2,3}$ \\ ${ }^{1}$ Students' Scientific Circle of Travel Medicine, Medical University of Warsaw, Poland \\ ${ }^{2}$ Department of Epidemiology and Tropical Medicine; Military Institute of Medicine, Warsaw, Poland \\ ${ }^{3}$ Department of Tropical Medicine and Epidemiology, Institute of Maritime and Tropical Medicine, \\ Medical University of Gdansk, Poland
}

\begin{abstract}
Thyroid disorders account for a majority of endocrine diseases. The most frequent among them are Hashimoto's thyroiditis, thyroid nodules and cancer with hyperthyroidism or hypothyroidism. Many thyroid patients travel a lot and therefore require appropriate guidance from their doctor. The aim of the review article is to discuss various aspects of travel in order to determine the optimal travel conditions for thyroid patients. Thyroid travelers must be well prepared for their journey. They should put particular emphasis on the choice of destination, the season and iodine resources at the planned place of stay. Before going on a journey, they are advised to check their health insurance, buy enough medications, prepare a copy of prescriptions and all other necessary documents. Depending on the means of transport, a few precautions should be taken to avoid infections or worsening of thyroid symptoms during travel. To deal with unpredictable events which might occur at the place of stay, travelers are recommended to take a first-aid kit containing basic medications. It needs to be stressed that many drugs are responsible for thyroid function disruptions and should only be used after consultation with a healthcare professional. Avoiding stress, maintaining good night's rest, as well as following a healthy diet, are all of great importance in managing thyroid diseases. Observing a few simple rules can minimize the frequency of flare-ups and the occurrence of the life-threatening thyroid storm. Traveling is an inseparable element of life for many people. Therefore, ensuring that patients are well informed about how to travel safely may prevent unwanted health events during travel.
\end{abstract}

(Int Marit Health 2021; 72, 4: 293-303)

Key words: travelers, thyroid disorders, epidemiology

\section{INTRODUCTION}

Thyroid gland is a vital organ situated anteriorly in the lower neck and laterally to the thyroid cartilage. It consists of two lateral lobes connected by isthmus. Thyroid is one of the biggest endocrine glands and it is a richly vascular organ [1]. In up to $44.6 \%$ of the general population a third thyroid lobe exists, also known as the pyramidal lobe, which is a persistent embryonic remnant of the thyroglossal duct [2-4]. Along its path functional accessory thyroid glands may also be found [2]. Thyroid gland is built up of lobules, which are divided into thyroid follicles, the structural and functional units of the thyroid gland. Each follicle is filled with fluid containing thyroglobulin, which is the matrix for the synthesis of thyroid hormones occurring by tyrosine iodination. As a result, triiodothyronine (T3) and tetraiodothyronine (T4) are produced. Thyroid is the only endocrine gland which is capable of storing enough of its hormones to cover a 10-month demand of the body [5]. Hence any disruptive factors or pharmacologic actions require time to result in the change of the thyroid gland function. Furthermore, it enables adaptation to iodine nutritional undersupply [6, 7]. T4:T3 secreting ratio is approximately 14:1 on average. Tetraiodothyronine is often considered as a 'prohormone', as $80 \%$ of T4 is converted peripherally into the T3 and reverse T3 (rT3). T3 is the active form of thyroid hormones with 20-30 times higher affinity to

Prof. Krzysztof Korzeniewski, MD, PhD, Military Institute of Medicine, Head of the Department of Epidemiology and Tropical Medicine, Szaserów St. 128, 04-141 Warsaw, $\triangle$ Poland, e-mail: kkorzeniewski@wim.mil.pl; Medical University of Gdansk, Institute of Maritime and Tropical Medicine, Department of Tropical Medicine and Epidemiology,

Powstania Styczniowego St. 9B, 81-519 Gdynia, Poland, e-mail: kkorzeniewski@gumed.edu.pl

This article is available in open access under Creative Common Attribution-Non-Commercial-No Derivatives 4.0 International (CC BY-NC-ND 4.0) license, allowing to download articles and share them with others as long as they credit the authors and the publisher, but without permission to change them in any way or use them commercially. 
TRs than that of T4, having main effect on target cells [8]. Thyroid hormones exert their biological effect by binding to nuclear receptors. Their actions are necessary for normal metabolism of all the cells in the body, considering their permissive effect upon growth hormone and their cooperation with glucocorticoids and especially, catecholamines, by raising the sensitivity of the beta-adrenergic receptor, effecting heart rate and its contraction. They have a particularly strong effect on the central nervous system and the basal metabolic rate, by regulating carbohydrate metabolism. Moreover, thyroid hormones have a great impact on a person's mood and behavior, which is why thyroid gland disorders manifest in such a distinguishing manner $[9,10]$.

\section{EPIDEMIOLOGY}

Thyroid disorders are the most prevalent endocrine problems; many of them have an autoimmune basis; the statistics clearly show that the incidence of such disorders is remarkably higher in female patients [11]. Thyroid abnormalities may be classified into the following categories: thyroid disorders - hyperthyroidism, hypothyroidism and diseases of the gland - thyroid nodules, goiter and cancer. Hyperthyroidism is the most common form of thyrotoxicosis, affecting $0.5-2 \%$ of women. Its common cause is the Graves' disease or toxinodular goiter. Graves' disease is of autoimmune origin. Symptoms result from increased metabolic rate and enhanced sympathetic activity, mainly manifesting as tachycardia, arrhythmia, increased perspiration and weight loss. The characteristic signs and symptoms of the Graves' disease also include ophthalmopathy and pretibial myxedema. Subclinical hyperthyroidism is also quite common in the general population, with the prevalence of $3 \%$ in people over 65 years [12]. Hypothyroidism is a condition of insufficient production of the thyroid hormones, which may be a result of thyroidectomy or autoimmune destruction of the gland. The annual incidence of spontaneous hypothyroidism was estimated at 3.5 cases per 1000 women and 0.6 cases per 1000 men. In low iodine areas the incidence rises to $1-2 \%$. Subclinical form of the disease was found to affect about $8 \%$ of women and $3 \%$ of men in the general population. Clinically, hypothyroidism manifests with myxedema coma with low blood pressure, fatigue, exercise intolerance, apathy, poor concentration; the symptoms may mimic depression [13]. Hashimoto's thyroiditis is another autoimmune disorder which is caused by gradual destruction of the gland. The incidence is considered to be 3.5 cases per 1000 women and 0.8 cases per 1000 men annually [13]. Transient thyrotoxicosis may occur during the initial stage of the disease (hashitoxicosis). The main signs and symptoms of Hashimoto's disease include painless enlargement of the gland, problems getting pregnant or any other symptoms specific for hypothyroidism [14]. Thyroid nodules are found in $6.4 \%$ of women and $1.5 \%$ of men over 60 years [12]. The most clinically relevant are the autonomous ones, which may be a part of multinodular toxic goiter. Initially they may be inactive because of insufficient iodine supply; however, their presence may be unmasked after uptake of iodine under various circumstances. Given the fact that elderly persons are generally more likely to have nodular goiter, they are at a much higher risk of developing thyrotoxicosis induced by iodization. Although thyroid cancer accounts for $>90 \%$ of all endocrine cancers, it is a rare malignancy. It accounts for $<1 \%$ of all malignancies in the United Kingdom [12]. The annual incidence in this country is 3.5 cases per 100,000 women and 1.3 cases per 100,000 men. Thyroid cancer manifests as a painless solitary thyroid nodule or goiter [7]. It seldom causes disruption in thyroid function, but it frequently results in compression or even displacement of the adjacent neck structures [7].

\section{RISK FACTORS RELATING TO THYROID DISORDERS}

Different thyroid disorders are relatively frequent in travelers, especially in certain age groups. Functionally they may be normo-, hypo- or hyperthyroid causing a large variety of pathological conditions resulting from multiple trigger factors. Thus, the risk for such travelers may vary from relatively low to critical. The role of healthcare professionals is not only to provide treatment to their patients but also to ensure their patients' wellbeing. For many people travel is an important element of their life. To fulfil this responsibility patients with thyroid conditions have to be informed about the necessary precautions they will need to take in order to minimize the risk of potential health problems. Careful planning helps anticipate potential risk factors which may occur during travel.

Climatic conditions. Temperature, climate and altitude were found to have a significant impact on the thyroid gland function: thyroid-stimulating hormone (TSH) and FT3 values increase in the winter but drop during the hot seasons. Patients with hyperthyroidism are recommended to avoid destinations with hot and humid weather; instead, because of their heat intolerance, they are advised to travel during colder seasons. Humid weather may be unbearable for hyperthyroid patients, as they already experience increased perspiration. In contrast, hypothyroid patients are advised not to travel to extremely cold destinations, as an underactive thyroid gland is incapable of maintaining proper thermoregulation. Travel to tropical countries is only possible when thyroid function is well controlled and a patient's condition is stabilized. Moreover, adequate precautions should be taken when going to places characterized by extremely dry climate, as it may aggravate mucosal dryness and lead to nasal bleeding. Another important risk factor with respect 
to climate is staying at the altitudes of over 5,400 $\mathrm{m}$ above sea level, where hypoxia occurs and thus the levels of thyroid hormones along with TSH are likely to increase. Hypoxic conditions may be challenging for thyroid patients, and therefore additional oxygen may be recommended. If thyroid patients undertake such an expedition, they should undergo gradual acclimatization to high-altitude conditions [15].

Low iodine-areas. The Earth's environment is rich in iodine, although its resources are not evenly distributed; the highest iodine concentration is in coastal regions, whereas soils of inlands, mountainous areas, grounds that are recurrently flooded or even some of the coasts are low in iodine. The areas which are low in iodine include: the mountainous parts of Europe (Germany, Austria, Switzerland), the Songkhala valley and the mountain regions in China, the northern parts of India and the Ganges Valley, the Irrawaddy Valley in Burma, the Andes in South America and some parts of Africa to a lesser extent [16]. Despite intensive efforts to restore iodine in affected countries by universal salt iodization, which is by now the most cost-effective strategy, some areas are still at high risk of iodine deficiency [17]. The daily dose of iodine recommended by the World Health Organization (WHO) is $150 \mu \mathrm{g}$. If a traveler is staying in a low-iodine area for a longer period, they should regularly undergo thyroid function tests in order to check whether the iodine supply is sufficient or if iodine supplementation should be commenced. Unfortunately, due to lack of data it is difficult to estimate how long a traveler can stay in low-iodine place, without developing symptoms of deficiency, because it is highly individual and depends on many factors. For blood testing purpose patients may send a serum sample in brown monovette tube to the institution making the examination in their country. Blood work arrangements should be made prior to a journey to make sure patients have got all the equipment needed for collecting and sending blood sample [11].

\section{TRAVEL}

Before travel, it is essential for patients to check their health insurance to ensure it provides enough coverage in the country of destination. Bearing in mind that unexpected situations may occur, such as delays, damage to the baggage or losing the medications, it will be reasonable to take excess amount of medication. It is not recommended to buy medications at the destination as there may be differences in the quality of the drugs. In case of a stay longer than a few months, a doctor may give a patient a prescription for an extra supply of medicines they take on a regular basis. For easier access, taking a photo of the prescription medications and saving it on an electrical device is always an option. If travel is associated with crossing multiple time zones it is recommended to talk to a physician about ad- justing the medication schedule. With regard to transport, especially air transport, thyroid patients are recommended to rebook their flight if they have an ear, nose or sinus infection. Flying with the abovementioned conditions can cause severe pain, bleeding and increases the risk of an eardrum rupture even if decongestants are used. Many airlines do not charge passengers for rescheduling or cancelling their booking on condition that they provide a certificate from their doctor explaining the need for rescheduling the flight. Travel first-aid kit should also contain medications for sunburn or insect bites. To avoid sunburns, sunscreen should be used whenever going outdoors. Photosensitivity can be caused by radiotherapy, chemotherapy or certain medications, including the most common tetracycline, doxycycline, voriconazole, amiodarone, hydrochlorothiazide, naproxen, piroxicam, chlorpromazine and thioridazine.

Travel by plane. Planes are a popular means of transport; however, high altitude exposure carries a degree of risk for thyroid patients. A pre-travel consultation with a physician is strongly advised especially to patients after a surgery, including eye and dental procedures, those with pulmonary and cardiac diseases, or cancer patients as these patients are at a higher risk of complications. The conditions inside an aircraft cabin are unfavorable to human health. Aircrafts are flying at the altitudes of up to $12,000 \mathrm{~m}$ above sea level $(39,000 \mathrm{ft})$. A cabin is pressurized to an equivalent of the atmospheric pressure at an altitude of 1,500-2,400 m above sea level $(5,000-8,000 \mathrm{ft})$ to maintain partial pressure of oxygen. Nevertheless, Humphreys et al. [18] found that both short and long-haul flights decrease oxygen saturation $\left(\mathrm{SpO}_{2}\right)$ from $97 \%$ at the ground level to $93 \%$ at cruising altitude in both healthy patients and those with comorbidities. Values of $\mathrm{SpO}_{2}$ below $94 \%$ are an indication for applying supplementary oxygen [18]. It needs to be stressed that patients suffering from chronic hypoxia may experience an even greater reduction in $\mathrm{SpO}_{2}$ when exposed to high altitudes. It is a meaningful discovery when it comes to thyroid patients; those with hyperthyroidism, for instance, have increased demand for oxygen. Moreover, hypoxemia activates the sympathetic nervous system, which causes increased heart rate and may increase the risk of angina and dysrhythmias. These effects are especially unwanted for patients with hyperthyroidism, as they already experience similar symptoms [18]. Anemia is another indication for the administration of additional oxygen. Long-haul flights are associated with immobility and dehydration. These factors reduce blood flow in lower extremities, which may lead to local hypoxia resulting in vasodilation and increased capillary permeability. This is how limb edema starts to develop [18]. To minimize the risk of its occurrence, passengers are advised to wear loose-fitting clothes and footwear which can be easily taken off. Another tip is to leave a seat and 
walk around every hour or two and stretch the legs, neck and shoulders every $30 \mathrm{~min}$. Passengers must remember to stand up slowly to avoid vertigo. Walking and stretching the muscles will help to relieve muscle and joint pain, which often affects thyroid patients. It may also prevent the formation of blood clots, as the blood that pooled in the extremities while a person was immobilized is forced to circulate [11]. Deep vein thrombosis (DVT) is a well-known complication of long-haul flights; hence passengers are advised to wear antiembolic stockings. Sometimes taking aspirin or low molecular weight heparin is recommended. Individuals susceptible to edema should avoid wearing rings or wristwatches during a flight. According to the available data, humidity in an aircraft cabin is below $20 \%$, while at the ground level it is significantly above $30 \%$. Low humidity conditions cause dehydration, so drinking fluids ( $250 \mathrm{~mL}$ every hour) throughout the flight is recommended, preferably bottled water as a safety precaution against infectious diseases. Alcohol consumption should be reduced 24 hours before a flight. Other dehydrating factors including coffee, soda and salty snacks should be avoided. Staying hydrated is essential to maintain proper peristalsis. Constipation is common in people with hypothyroidism, and steatorrhea is prevalent among hyperthyroid patients. Low humidity aggravates dry skin, dryness of nasal mucosa and eyes; the conditions can be easily managed by the use of moisturizers, saline nasal spray, eye drops and by choosing to wear glasses instead of contact lenses [11]. Staying on board with other passengers during long-haul flights carries a high risk of contracting an infectious disease. To reduce the risk of becoming ill it is important to order bottled or canned beverages only and to avoid drinking airline-provided coffee and tea, as the United States Environmental Protection Agency proved that airline water could be contaminated by bacteria [19]. Multiple inflight food-borne outbreaks have been reported over the years, among which the most common were those caused by Salmonella, Staphylococcus, viral-induced enteritis and Norwalk-like agent, what was associated with mortality [20]. Thyroid patients already experience abnormal peristalsis, hence additional disruption in the intestines function may lead to serious complications, e.g., dehydration. Hashimoto's thyroiditis and Graves' disease can both be caused by infectious agents and make patients more susceptible to infections. Thyroid patients, in particular those with symptoms of hypothyroidism, suffer from cold intolerance, which may be inconvenient during travel. Patients need to stay cautious and not use blankets provided by the airline, because even if they are sealed they are infrequently disinfected. For those who are more sensitive to cold temperatures, dressing in layers is a good idea [11]. It is also recommended to wear a protective antimicrobial face mask in case there are sick passengers on board. Using disinfectant wipes to clean seatbelts, armrests and especially tray tables, which are considered to be a prime source of bacteria on a plane is always an option. Although, they are not legally required, most aircrafts are equipped with high efficiency particulate air filters (HEPA). They effectively filter air from dust, vapors, bacteria, fungi and viruses. Unfortunately, it seems not to be enough as there are at least several studies that reported of the spread of airborne infections such as tuberculosis, SARS, influenza and meningococcal disease aboard a plane. Novel pathogens can potentially cause outbreaks, as was the case in 2002 when acute respiratory syndrome was rapidly spread during air travel. The risk of developing an infection is not only limited to the aircraft cabin itself but it also exists at the airports, i.e., before and after a flight. The best example of an airport disease is the so-called airport malaria that occurs in and around airports all over the world. There is evidence that mosquitoes which are able to transmit malaria can be imported to non-endemic regions aboard planes. Out of 87 reported cases of airport malaria, 75 occurred in Europe. Dengue and yellow fever were also reported in European countries [20]. Hence, thyroid patients are advised to maintain good hand hygiene and wear a face mask while traveling by plane, as becoming ill during a journey may aggravate their underlying condition. Furthermore, many medications, such as antimalarial drugs have been reported to interfere with thyroid medications, thus potentially placing thyroid patients in a life-threatening situation while abroad [21]. Stress and fatigue are the main contributors to becoming ill, so travelers should remember that a good night rest and staying hydrated are crucial for sustaining immunity.

Patients with chronic diseases must not forget to take their medications when going on a journey; they should be kept in carry-on baggage. Furthermore, medicines should be kept in original packaging in case of a security check. Liquid medications are not always required to be put in a zip-top bag, but travelers are accountable for informing security about it so the medication can be screened separately [11].

Travel by boat. Before a boat or a ship journey it is a good idea to ask a physician for anticholinergic prescription drugs, e.g., scopolamine patches, to treat sea sickness. Hyperthyroidism itself can cause nausea and vomiting. Scopolamine, also known as hyoscine, works for up to 3 days, but should be administered 6 to 8 hours before travel. Another medication used to prevent motion sickness is dimenhydrinate, with administration time 0.5 to 1 hour before the start of the journey. Its combination with cinnarizine was reported to have a response rate of $78 \%$, although their effect lasts for 4-8 hours and their use should be limited to acute cases only. Alternatively, Sea-Band wristbands may be applied as a preventive measure. They work by stimulating the P6 acupressure point located on the anterior surface of the forearm 
and are believed to affect serotonin transmission. Although Sea-Bands' effectiveness is debatable, taking into consideration that they are a non-invasive and inexpensive form of preventing sea sickness it is worth giving them a chance, as they have proved to be effective in alleviating nausea and vomiting in pregnancy and after chemotherapy [22]. Natural supplements containing gingers are often used by patients as an unconventional antiemetic. High doses of vitamin $\mathrm{C}$ are also reported to be effective. Non-pharmacological interventions include transcutaneous electrical nerve stimulation and stress managing techniques, e.g., relaxing music or odors. Another point worth mentioning is the fact that travelers should avoid food and drinks, whose process of production is based on fermentation or require the use of microorganisms, such as tuna, cheese, salami, sauerkraut and red wine. These products are rich in histamine and eliminating them during the sea travel is a known preventive measure [23]. The antiemetic medications that are contradicted in treating motion sickness include metoclopramide, because it is known to cause extrapyramidal symptoms and QT prolongation, ondansetron and aprepitant, which cause headache and constipation.

Radioactive iodine. Both air travel and sea travel require security check. Patients who have recently undergone radioactive treatment may activate radiation detector. It is difficult to decide how long such an inconvenience may last, as it will depend on the rate of renal clearance of radioiodine [24]. It is speculated that patients may trigger airport radiation detectors for up to 95 days after the therapy. Therefore, patients are recommended to carry a certificate confirming they have had radioactive iodine treatment, in case they activate radiation detector and need to explain the situation to the airport staff [11].

\section{STRESS AND SLEEP}

Traveling is associated with a number of stressors. Therefore, travel should be planned in details in order to avoid stressful situations. Travelers are advised to arrive at airports in good time, book their hotel rooms well in advance and carefully plan their itinerary. It is crucial to bear in mind that stress may be both emotional or physical tension. Long journeys, lack of sleep, strenuous exercise and permanent tension also put strain on the body.

While it is challenging to maintain regular sleep hours on a journey, the best advice for thyroid patients is to prepare a realistic plan of the journey and make sure there is enough time for rest and meals. Sleep deprivation affects the immune system and increases the risk of autoimmune diseases [25]. The endocrine system is extremely vulnerable to circadian rhythm disruptions. The thyroid gland is driven by TSH changes, affected by sleep alterations. It was reported that acute, extreme sleep deprivation elevates
TSH secretion, while long-term but moderate lack of sleep decrease its release. There are studies which indicate that less than 7 hours of sleep or more than 9-10 hours of sleep are associated with a higher risk of subclinical thyroid dysfunctions. Hence, 7-8 hours are considered as normal sleep duration, which is associated with the lowest risk of TSH secretion alterations [26]. Maintaining the above mentioned sleep schedule may be challenging given the fact of changing time zones. For some patients it may be beneficial to try melatonin. It facilitates the adjustment of the internal body clock to the local time zone. The general advice is to take it about 30-60 minutes before going to bed [27]. When traveling east it is also advised to take it for 2 consecutive days prior to travel when the time at the destination is 11 p.m., because it takes longer to reset the circadian rhythm when traveling eastward. To reduce the risk of jet lag, travelers are recommended to stay awake during the flight when traveling east, and to try to have a rest while flying west. Stress increases secretion of glucocorticoids as well as catecholamines, resulting in tachycardia, tachypnea and elevated blood pressure. Hyperthyroidism often manifests with similar symptoms, which are normally treated with beta-blockers. Every stressful situation may aggravate a patient's condition by exacerbating the symptoms. Any kind of stress is associated with the risk of flare-ups of autoimmune disease and with thyroid disorders there is no difference. Despite crediting stress a suppressive function, its main influence on homeostasis is by shifting $T$ helper cells from Th1 toward a Th2, thus suppressing cellular immunity and enhancing humoral immunity. Bearing in mind that Graves' disease is characterized by lymphocytic infiltration of CD4+ Th2 cells, there is strong evidence that stress influences clinical expression of Graves' disease, increasing relapse rate, thus elevating anti-TSH antibodies. Moreover, during stress corticotrophin-releasing hormone is secreted in peripheral tissues, which results in the release of histamine - a wellknown trigger of acute inflammation. These destructive features of stress are reversible after stress withdrawal or palliated by glutathione intake, because of its ability to bring back antioxidant storage. It is widely used by patients with autoimmune thyroid disease, such as Graves' disease or Hashimoto's thyroiditis [28].

\section{DIET}

Autoimmune diseases, especially Hashimoto's thyroiditis, require self-care, as even minor triggers may be debilitating and result in flare-ups. Factors like menstruation, hormonal imbalance, viral or bacterial infections, excessive physical strain and nutritional deficiencies have considerable impact on the already impaired immune system. Avoiding the abovementioned risk factors and taking care of a physical and mental health are a priority. 
First of all, travelers should carefully plan when and where they are going to have their meals. Before going on a vacation they could search online for restaurants that offer healthy food. If possible, they might take their own kitchen equipment and food products to prepare their own meals. It's worth checking if there is a refrigerator at the accommodation - this will enable patients to keep the food fresh. When traveler is spending the whole day outdoors, and have no possibility of ordering a meal, it is a good idea to take along some packed snacks like crackers, energy bars or previously prepared meals, including, for example olives, sardines, celery or beef jerky [11].

The general recommendation for Hashimoto's disease patients is to eat 4-5 well balanced meals a day. Daily protein consumption should be up to $15-20 \%$ or even $25 \%$ of daily calorie intake. Sugar-free diet should be followed, particularly by patients with coexisting insulin resistance or diabetes. Increased blood glucose leads to an inflammatory response, which disrupts many bodily processes. Whole grains should be the main source of carbohydrates, as these are rich in dietary fiber, which is essential in maintaining balance in intestinal microbiota and bowel movements (the disorder of which is a common issue in hypothyroid patients). The minimum intake of fiber should be $25 \mathrm{~g}$ per day. To nourish the immune system, patients are recommended to eat sufficient amounts of fruit and vegetables and reduce the intake of processed food like sweets and chilled beverages. The use of pre-, pro- or synbiotics is also beneficial in thyroid patients. Intake of saturated fatty acids should be reduced, in favor of food containing omega-3 acids, in the amount of 1-2 g/day. Olive oil, avocado oil and walnut oil are excellent sources of fats [29]. Diet of patients with autoimmune thyroid disease, should be rich in iodine, iron, zinc, copper, magnesium, potassium, vitamins, $A, C, D$ and B group, as these elements were found to be in deficiency among these patients. Iron is profusely found in meat, animal offal and cocoa and plays an important role in the production of thyroid hormones. Magnesium and zinc, both found in pumpkin seeds, nuts and cocoa are responsible for decreasing antibody titers. Additionally, magnesium has anti-inflammatory properties, by reducing C-reactive protein. Vitamin D3, often overlooked, is responsible for maintaining the physiological functions of the immune system by activating or suppressing about 200-500 genes. This is why a number of studies presented clinical improvement in patients with autoimmune disease, when supplementation was introduced [29]. According to Mansournia et al. [30], an increase in vitamin D3 intake by $5 \mathrm{ng} / \mathrm{mL}$ reduces the risk of developing Hashimoto's disease by $19 \%$. Selenium is another essential mineral; it is a potent antioxidant and plays a protective role on the thyroid gland, when iodine is given in excess. Moreover, it suppresses secretion of inflammatory cytokines by T lymphocytes. A diverse diet is required for maintaining proper amount of vitamins, micro- and macroelements; hence supplementation in the form of capsules is needed only when nutritional changes do not bring a positive effect, as in the case of selenium deficiency, where studies showed that eating habits had no significant impact on selenium level in the blood [29]. The need of supplementation should be assessed based on symptoms of a particular element deficit, e.g., hair loss in zinc deficiency or upon blood test results, e.g., vitamin D3 and selenium, as excess of microelements are also harmful.

As many as $75.9 \%$ of patients with Hashimoto's thyroiditis suffer from lactose intolerance; hence products containing lactose should be eliminated from their diet, after positive diagnostic tests. It's essential information as not observing the restrictions, while having lactose intolerance, causes a decrease in the bioavailability of levothyroxine, and thus patients will require higher doses of the medication [29, 31, 32]. Similarly, gluten-free diet should be observed, when there is coexistence of celiac disease or non-celiac gluten intolerance, which are significantly more prevalent in Hashimoto's disease. The reason for treating co-existing celiac disease is because of cross-reactions between gliadin and thyroid antigens and the possibility of immune system attacking the body's tissues. Screening for celiac disease should be considered among patients, who have difficulty achieving sufficient nutrition and regulating the concentration of thyroid hormones [29]. According to 1 study, patients without the clinical symptoms of celiac disease, but with the presence of anti-tissue transglutaminase antibodies, achieved better results after they had gone on an elimination diet, than control group. A significant reduction in the level of antibodies against thyroid peroxidase and thyroglobulin was reported [33]. Avoiding food containing gluten before initiating diagnostic process may result in false test results [29]. Diet should be introduced only after positive diagnostic tests, according to the Expert Committee of the Section of Medical Dietetics of the Polish Society for Parental, Enteral Nutrition and Metabolism (POLSPEN) [34]. There is a lack of studies proving benefits of introducing gluten-free diet in patients without co-occurring celiac disease or another form of gluten intolerance. Furthermore, if patients put themselves on elimination diet without medical indications, they increase the risk of harmful health consequences, such as an increased cardiovascular risk or nutritional deficiencies. Whole grains and unprocessed cereals contain more fiber and vitamins. That is why after introducing gluten-free diet, patient should stay under a care of a dietician to avoid iron, folate, vitamin B12, D3, magnesium and selenium deficiency, all significant in maintaining the proper functions of the immune system and the thyroid [29]. 
Hyperthyroid patients should also bear in mind, that diet supports their health and can alleviate many symptoms. Because of increased metabolic rate it is recommended that calorie intake should be upped by $15-25 \%$ or even by up to $50-80 \%$, depending on how severely thyroid gland is overactive. Protein supply should be maintained at about 110-130 g per day. Products containing fats and processed food should be avoided, as well as caffeine, which causes insomnia and restlessness, exacerbating symptoms patient with hyperthyroidism already face. In case of gastrointestinal issues, e.g., diarrhea, easily digestible diet should be introduced [35].

\section{ALLERGIES}

Allergic rhinitis has been found to be more prevalent among patients with Hashimoto's thyroiditis and Graves' disease. Hence, avoidance of allergens is critical so as not to stimulate the immune system. Environmental antigens not only induce local allergic reactions, but also aggravate autoimmune conditions [36-38]. Dust, feather pillows, synthetic scents are among the common triggers. Allergy sufferers may be advised to track pollen counts and stay indoors as much as possible during times when pollen counts reach high levels. Cetirizine, fexofenadine, levocetirizine, loratadine which are widely available in supermarkets are highly effective for allergic rhinitis. Travelers with allergies are recommended to book hotels which offer allergy-friendly rooms with air purifiers, fragrance-free cosmetics and windows that can be opened to ventilate the room. Choosing hypoallergenic products is recommended. Many allergies are manifested by skin redness and in some cases hives. The food which a patient is sensitive to should also be eliminated from his/her diet [11].

\section{DRUG INTERACTIONS}

Levothyroxine should be taken orally on an empty stomach as food and many other medications, e.g., calcium, iron and aluminum-containing supplements, bisphosphonates inhibiting bone resorption, sulfonylurea derivatives used in the treatment of diabetes and proton pump inhibitors (antacids), sucralfate to treat stomach conditions and also drugs lowering the level of cholesterol; e.g., resin binders affect the absorption of thyroid hormones. Cholesterol lowering statins, dopamine agonists, estrogens, androgens and tamoxifen, all influence thyroid function tests. Ipodate, iopanoic acid and tyropanoate and contrast media inhibit conversion of T4 to T3, causing transient rise in TSH level. Antiepileptic drugs like carbamazepine or phenytoin enhance levothyroxine clearance, similarly to sertraline. Lithium carbonate, a normotymic drug, causes subclinical hypothyroidism as the compound concentrates in the gland and disrupts hormone synthesis at the same time increasing the risk of the rise in the levels of antibodies, particularly in female patients.
Amiodarone, a drug used to manage atrial fibrillation and congestive heart failure, is known for its great impact on thyroid function. Up to $24 \%$ of patients taking this medication suffer from amiodarone-induced thyroid disorder. Hypothyroidism is more common than hyperthyroidism and is recognized with difficulty, as bradycardia and constipation are both listed among the possible side effect of amiodarone. Overactive thyroid gland may present as Graves' disease-like hyperthyroidism with the presence of antibodies or subacute thyroiditis with no antithyroid antibodies detected. Treatment is undeniably needed, even after amiodarone discontinuation, because of drug's accumulation in adipose tissue.

Glucocorticoids are widely used in numerous dermatological, ophthalmological and allergic conditions as well as suppressive therapy. Their impact is mainly by decreasing TSH level, and in large doses reducing T3 level, to prevent thyroid storm in hyperthyroidism.

Furosemide, first-line therapy in lowering acute surge in blood pressure, salicylates and non-steroidal anti-inflammatory drugs (NSAIDs), such as diclofenac or naproxen, transiently increase free T4. During prolonged use of NSAIDs hormones return to normal.

Beta-blockers, including propranolol, atenolol, metoprolol, used to maintain normal heart rate, suppresses peripheral conversion of T4 to T3.

Rifampicin is an antibiotic used to prevent infections caused by meningococci and Haemophilus influenzae type $b$. It is used for treating tuberculosis and leprosy. It enhances T4 metabolism by inducing hepatic enzymes. Hence, maintenance of euthyroidism will require higher doses of thyroxine.

Interferon alpha, used as a long-term treatment for hepatitis $\mathrm{C}$, has also been proved to cause hypothyroidism, as well as hyperthyroidism. The onset may be as early as 6-8 weeks of the therapy course, albeit these conditions are transient and treatment is only symptomatic [39].

Antimalarial chloroquine and proguanil were reported to stimulate TSH level in hypothyroid female patient receiving thyroxine sodium. The effect on thyroid gland was observed after 4 weeks of starting medication. It is contentious whether any of these drugs directly affect hypothalamus. It is much more likely that antimalarial drugs enhance thyroxine clearance by activating liver enzymes [21].

Metoclopramide should not be used by thyroid patients to suppress nausea and vomiting as it slightly elevates TSH level in function tests. Also, it is not advised to be taken to prevent motion sickness.

lodides, prevalent in numerous medications such as povidone iodine, iodinated glycerol, tablets containing seaweed, herbal preparations and dietary supplements, expose patients to an iodine load which can lead to thyroid dysfunction. Jod-Basedow disease is iodide-induced hyperthyroidism, which occurs exclusively in patients with endemic 
goiter, Graves' disease, toxic multinodular goiter and thyroid adenomas. It usually develops within 3-8 weeks of increased exposure and cannot be suppressed by radioactive iodine, because iodine loading prevents the Wolff-Chaikoff block. Beta-blockers and thioamides are often required to be taken for several months. lodide-induced hypothyroidism also occurs when the gland is not able to overcome the Wolff-Chaikoff block. Thyroxine therapy should then be commenced [39]. It is crucial for patients to be aware of the iodine contents in the products they use. At present, cough or expectorant preparations do not contain iodine. Among newer medications only Waterbury's Compound was found to contain sodium iodide [40]. Thyroid patients are strongly recommended to check the iodine contents in all the medications they are using as thyroid malfunction was even reported after the use of povidone iodine locally for vaginal irritation [39].

\section{IODIZATION OF WATER}

The matter of water quality is extremely important for those traveling to parts of the world where the available water supplies are non-potable. lodine is a widely accessible, effective and low-cost method of water disinfection. Albeit, its use entails the risk of thyroid function disruption, because of excess iodine load. There are several types of iodine solutions e.g. tincture, povidone, Lugol's and saturated aqueous solutions with iodine crystals. Tablets and iodine resins are also a popular option among travelers. The latter may be considered as the most convenient form, as the residual iodine concentration is significantly lower, the solution is much more stable and the equipment provides two additional methods of eliminating microorganisms - microfiltration of Cryptosporidium oocysts and granular activated charcoal, with the aim of filtering the residual iodine in water. lodine-induced hypothyroidism is much more common and may develop in patients with or without underlying thyroid disease. lodine-induced hyperthyroidism also occurs, particularly in thyroid patients or persons with previous iodine deficiency. These patients exhibit a higher rate of multi nodular goiter with autonomous nodules, which in case of an increased iodine load are no longer suppressed by iodine deficiency. Because a safe daily dose of iodine has not been unambiguously determined, WHO only recommends short-term and emergency use of iodine or chlorine-based products in water treatment. Furthermore, thyroid function tests should be conducted before and during water treatment [41].

\section{VACCINATIONS}

The crucial part of travel planning is to consider if there is an indication for pre-travel vaccination. The need of immunization is based upon country of destination, a patient's health history and behaviors related to travel, e.g., type of accommodation, food and spending time outdoors. It is recommended that travelers get fully vaccinated in their homeland before travel, as patients may encounter difficulties in completing their course abroad because of uncertain vaccine quality as well the risk of as using unsafe (infected) needles. Details about an exact trip itinerary and dosing should always be discussed individually with a physician. In this review, only the most prevalent vaccines will be discussed.

Vaccine against tetanus-diphtheria-acellular pertussis should be administered in every adult patient who did not receive a booster. Traveling to the remote countries is a strong indication for getting boosters at 10-year intervals.

Vaccine against varicella is recommended for adult patients without the evidence of immunity against this disease (2 doses at least 6 weeks apart).

As respiratory diseases are easy to catch during travel, all healthy travelers above 65 years should be immunized with a pneumococcal vaccine (1 dose; 13 serotypes of Streptococcus pneumoniae).

Adults should receive a lifetime total of at least 2 doses of vaccine against measles-mumps-rubella or be able to present a written confirmation that they have recorded from diseases and acquired natural immunity.

Vaccination against hepatitis $B$ is indicated for all non-vaccinated patients. It is particularly important for adventure travelers ( 3 doses: 0, 1, 6 month).

Vaccine against influenza is especially important when staying on cruise ships or going to mass-gathering events. It is strongly advised for those visiting the tropics, regardless a time of the year and temperate countries where is currently winter. Due to high mutation rate of the influenza viruses, travelers are required to take the latest available vaccine. Its efficacy lasts for about 6-12 months. After that time revaccination should be considered. It is worth to stress that even if the immunization will not prevent a patient from falling ill, it will certainly protect against influenza complications.

Quadrivalent meningococcal vaccine is recommended for those going to the "meningitis belt" in Sub-Saharan Africa, during the dry season, lasting from December to June. One dose of the quadrivalent vaccine $\mathrm{A}, \mathrm{C}, \mathrm{W}-135, \mathrm{Y}$ provides lifetime immunity. Meningococcal $B$ vaccine is indicated for travelers with comorbid conditions, e.g., asplenia or complement deficiency.

Adults who received complete primary vaccine series against poliomyelitis in childhood and are traveling to risk countries (polio-endemic or reported cases of vaccine-derived polio) should be administered with one-time single dose of inactivated vaccine as a booster (every 10 years).

Vaccine against hepatitis $A$ is indicated for every non-immune traveler going to countries other than United 
States, Canada, Japan, Australia, New Zealand, and developed countries in Europe. Two doses given 6-12 months apart provides long-term protection. Patients with a history of hepatitis or those who have lived in an endemic country may be tested for antibodies. When the patient cannot be vaccinated they will benefit from intramuscular immunoglobulin dosing. Non-immune travelers who experienced hepatitis A exposure are given a single dose of vaccine or immunoglobulin, if available, for immunocompromised patients.

Vaccine against typhoid fever is indicated when going to the Indian subcontinent or to endemic areas in Africa. However, food and water precautions should still be taken, as vaccine is only $53-72 \%$ protective.

Indication for vaccine against yellow fever is limited to the individuals at risk, as serious vaccine-associated adverse effects were reported. The decision about vaccination should be based upon the risk of transmission in this area and country entry requirements. That is why up-to-date maps and national laws should be checked by traveler.

Vaccine against rabies is recommended when long-staying in endemic areas of Latin America, Asia or Africa. Receiving it does not eliminate the need for vaccination after an exposure but it simplifies the procedure. Immunocompromised patients should get advice from specialized travel clinic before a journey. Live vaccine administration should be delayed 1 month after immunosuppressive corticosteroids are withdrawn. For chemotherapy and radiation therapy the waiting period is 3 months. Pneumococcal as well as hepatitis $B$ vaccine high-dose regimen is recommended for all immunocompromised travelers. Moreover, titers should be evaluated after vaccination to determine the antibody response [42].

\section{ADVICE FOR PATIENTS WITH THYROID CANCER}

Recently, traveling has become easier and more available to cancer patients, which is due to better quality of their life. Although travel is not impossible for such patients, there are precautions that should be taken before they decide to travel. Cancer patients are recommended to take a written summary of their medical history, as well as contact details of their attending physician. The documents are advised to be translated into the language of the country the patient is going to visit. Travel first-aid kit should contain enough amount of oral cancer medications, as well as other drugs alleviating the common cancer symptoms, such as antiemetics, antidiarrheals and analgesics. In terms of transporting the medication, a patient should check the current regulations regarding the transport of medications in the country of destination, to know exactly which medication and in what quantity can be brought into a given country. Some coun- tries may have strict restrictions on the transport of opioid medications. Cancer patients should never forget about the sunscreen, as they often experience photosensitivity after systemic treatment. Vaccinations should be discussed with a physician well in advance; travel destination and current treatment plan of the patient should be taken into account when considering if there is a need for vaccination. Vaccines should be avoided in cases of considerable exogenous immunosuppression, because patients undergoing immunosupressive treatment are less responsive to vaccinations. That is the reason why it is recommended to start all vaccinations 2-3 months before a journey, it will ensure enough time to perform serologic evaluation, confirming protective level of antibodies. Particular emphasis should be put on preventing infections. Observing simple rules of hygiene may not be enough and patients are strongly advised to avoid traveling during peak time of neutropenia while receiving chemotherapy.

Generally, patients should be clinically stable to be allowed to go on a journey. Moreover, undergoing radiation or systemic treatment is a strong contradiction against commercial air travel. Oxygen deficiency may lead to complications. That is why cancer patients are referred to perform hypoxic challenge test to determine the need for oxygen supplementation during a flight. In general, airlines recommend hemoglobin level above $8.5 \mathrm{~g} / \mathrm{dL}$. There were also reports of acute mountain sickness and high-altitude cerebral edema after head and neck surgery or radiation therapy.

Patients with active cancer are at a higher risk for venous thromboembolism. Before a flight, prophylaxis may be considered. Low molecular weight heparin is the usual drug of choice in such cases. Compression stockings are also advised on flights lasting longer than 5 hours. Other tips for decreasing the risk of blood clotting is maintaining hydration and doing some light exercises during the flight. Guidelines for patients with the history of DVT or pulmonary embolism include medical clearance within 21 days of the medical event. Patients should remain stable while taking anticoagulants. A history of DVT is an absolute contraindication to flying for 4 weeks after the event. Air travel is allowed after DVT is treated and if pre-exercise or postexercise desaturation does not occur. Postoperative or postirradiation lymphedema may origin or be exacerbated by pressure changes inside a plane cabin. It is contentious how much air travel increases the risk of it; nevertheless, it is recommended to wear loose-fitting clothes, shoes that can be easily taken of and avoid wearing rings and watches. As cancer patients have diminished physical performance, lymphedema may additionally make carrying heavy luggage difficult. To minimize the physical strain, airports offer assistance. Arrangements should be made early enough before travel [43]. 


\section{THYROID STORM}

Thyrotoxic crisis, also known as thyroid storm, develops in the course of hyperthyroidism. One hypothesis states that it occurs due to excessive secretion of thyroid hormones. This, however, is debatable and as yet the condition is of uncertain etiology. The condition may be caused by a number of factors, including the discontinuation of antithyroid medication, a trauma, a diabetic ketoacidosis, recent use of iodinated contrast medium or a medication interfering with thyroid function like amiodarone or salicylates. Acute infections also account for thyroid storm. Clinically, the condition is expressed by fever, tachycardia, arrhythmia, nausea, vomiting, diarrhea and central nervous system symptoms including agitation, delirium, anxiety, psychosis or coma. It is crucial that the condition is diagnosed at an early stage, as the mortality despite treatment achieves a rate of up to $25 \%$. Thyroid patients should be aware that the condition is life-threatening and its treatment requires professional care. Patients must be admitted to the intensive care unit where their heart function can be monitored. Along with supportive measures like IV fluids and oxygen, patients receive beta-blockers, thioamides, glucocorticoids, iodine solution and others. Patients should be informed how serious thyroid storm is and that in case of suspected thyroid storm visiting an emergency department should not be delayed [44].

\section{CONCLUSIONS}

Thyroid diseases are not a contraindication to travel. Thyroid patients can safely travel if they observe certain basic precautions. It is important to choose the destination and the season for traveling carefully, depending on the condition a patient suffers from. Climate has a significant impact on thyroid gland function. Hyperthyroid patients, for example, experience increased perspiration and have heat intolerance, whereas hypothyroid patients are extremely sensitive to cold temperatures. Humidity level and altitude should also be considered. Before travel, thyroid patients are advised to check if their health insurance provides medical coverage at their destination. Patients are recommended to consult with a physician which vaccinations they should receive before a journey. Travelers need to make sure they have an adequate supply of all the necessary medications in case their luggage is delayed, lost or damaged. The means of transport should also be chosen carefully, as travel, especially air travel, may aggravate certain underlying conditions. When traveling by plane, the administration of supplementary oxygen should be considered in patients suffering from chronic hypoxia. To prevent dehydration caused by low humidity on board a plane, travelers are advised to drink plenty of non-alcoholic beverages and use moisturizers. It is recommended to leave a seat and walk around every hour to restore the circulation of the blood and minimize the risk of blood clots formation. As staying on board with many other passengers carries a high risk of catching an infection, it is important to observe good hygiene practices and wear an antimicrobial face mask during the flight. Sea travel entails the risk of sea sickness, therefore, it is a good idea to ask a physician for anticholinergic prescription drugs prior to a journey. Thyroid patients must not forget to maintain a healthy lifestyle when they travel, this means avoiding stress, having a regular sleep schedule and following a balanced diet. A journey should be planned in details; it is important to arrive at an airport in good time, book a hotel room well in advance and choose the dining facilities carefully. Patients with thyroid cancer should also bear in mind adjusting their travel plan to their health condition and wellbeing. Thyroid patients can safely travel if only they follow some simple rules. Informing a patient about the standard precautionary measures and the steps which need to be taken in case of a medical emergency is a key to preventing life-threatening situations during travel and getting a thyroid patient enjoy their journey.

\section{Conflict of interest: None declared}

\section{REFERENCES}

1. Standring S, Borley NR, Gray H. Gray's anatomy: the anatomical basis of clinical practice. 40th Ed. Churchill Livingstone/Elsevier, Edinburgh 2008.

2. Mortensen C, Lockyer H, Loveday E. The incidence and morphological features of pyramidal lobe on thyroid ultrasound. Ultrasound. 2014; 22(4): 192-198, doi: 10.1177/1742271X14554677, indexed in Pubmed: 27433219.

3. Kim DW, Jung SoL, Baek JH, etal. The prevalence and features of thyroid pyramidal lobe, accessory thyroid, and ectopic thyroid as assessed by computed tomography: a multicenter study. Thyroid. 2013; 23(1): 84-91, doi: 10.1089/thy.2012.0253, indexed in Pubmed: 23031220.

4. Harjeet A, Sahni D, Jit I, et al. Shape, measurements and weight of the thyroid gland in northwest Indians. Surg Radiol Anat. 2004; 26(2): 91-95, doi: 10.1007/s00276-003-0194-y, indexed in Pubmed: 14615915.

5. Sawicki W, Malejczyk J. Histologia. Wydawnictwo Lekarskie PZWL, Warszawa 2012: 473-477.

6. Medeiros-Neto G. Thyroglobulin. In: Henry HL, Norman AW (eds). Encyclopedia of Hormones. Academic Press 2003: 448-457.

7. Khan YS, Farhana A. Histology, Thyroid Gland. In: StatPearls [Internet]. Treasure Island (FL): StatPearls Publishing; 2020 Jan.

8. Sandler B, Webb P, Apriletti JW, et al. Thyroxine-thyroid hormone receptor interactions. J Biol Chem. 2004; 279(53): 55801-55808, doi: 10.1074/jbc.M410124200, indexed in Pubmed: 15466465.

9. Leung A, Brent G. The Influence of Thyroid Hormone on Growth Hormone Secretion and Action. In: Cohen LE (Ed). Growth Hormone Deficiency. Springer International Publishing, Switzerland 2016: 29-46.

10. Ritter JM, Flower R, Henderson G, Loke YK, MacEwan D, Rang HP. Rang \& Dale's Pharmacology. Elsevier/Churchill Livingstone, Edinburgh 2020: 448-454.

11. Ghafari F, Izadi M, Hossein P. Traveling guides for patients suffering from thyroid disorders. Int J Travel Med Glob Health. 2015; 3(4): 155-159, doi: 10.20286/ijtmgh-0304146. 
12. Vanderpump M. The epidemiology of thyroid disease. Br Med Bull. 2011; 99(1): 39-51, doi: 10.1093/bmb/ldr030.

13. Maitra A. Endoctrine system. In: Kumar V, Abbas AK, Aster JC (Eds). Robbins Basic Pathology. 10th Ed. Elsevier Health Sciences Division 2017: 749-797.

14. Mincer DL, Jialal I. Hashimoto Thyroiditis. In: StatPearls [Internet]. Treasure Island (FL): StatPearls Publishing; 2020 Jan.

15. Mahwi TO, Abdulateef DS. Relation of different components of climate with human pituitary-thyroid axis and FT3/FT4 ratio: a study on euthyroid and $\mathrm{SCH}$ subjects in two different seasons. Int J Endocrinol. 2019; 2019: 2762978, doi: 10.1155/2019/2762978, indexed in Pubmed: 30800161.

16. Eastman CJ, Zimmermann MB. The lodine Deficiency Disorders. In: Feingold KR, Anawalt B, Boyce A, et al. (Eds). Endotext. South Dartmouth (MA): MDText.com, Inc. 2018. https://www.ncbi.nlm.nih. gov/books/NBK285556/.

17. Bonofiglio D, Catalano S. Effects of lodine Intake and Nutraceuticals in Thyroidology: Update and Prospects. Nutrients. 2020; 12(5), doi: 10.3390/nu12051491, indexed in Pubmed: 32443880.

18. Humphreys S, Deyermond R, Bali I, et al. The effect of high altitude commercial air travel on oxygen saturation. Anaesthesia. 2005; 60(5): 458-460, doi: 10.1111/j.1365-2044.2005.04124.x, indexed in Pubmed: 15819766.

19. Environmental Protection Agency. EPA Announces New Aircraft Drinking Water Quality Data. https://archive.epa.gov/epapages/ newsroom_archive/newsreleases/4cad7566ca567d3f85256f8e0053ce39.html (Accessed: 19 January 2005).

20. Mangili A, Gendreau MA. Transmission of infectious diseases during commercial air travel. Lancet. 2005; 365(9463): 989-996, doi: 10.1016/S0140-6736(05)71089-8, indexed in Pubmed: 15767002.

21. Munera Y, Hugues FC, Le Jeunne C, et al. Interaction of thyroxine sodium with antimalarial drugs. BMJ. 1997; 314(7094): 1593, doi: 10.1136/bmj.314.7094.1593, indexed in Pubmed: 9186170.

22. Lee EJ, Frazier SK. The efficacy of acupressure for symptom management: a systematic review. J Pain Symptom Manage. 2011; 42(4): 589-603, doi: 10.1016/j.jpainsymman.2011.01.007, indexed in Pubmed: 21531533.

23. Koch A, Cascorbi I, Westhofen M, et al. The neurophysiology and treatment of motion sickness. Dtsch Arztebl Int. 2018; 115(41): 687-696, doi: 10.3238/arztebl.2018.0687, indexed in Pubmed: 30406755 .

24. Cuthbertson DJ, Davidson J. What to tell patients about radioiodine therapy. BMJ. 2006; 333(7562): 271-272, doi: 10.1136/ bmj.333.7562.271, indexed in Pubmed: 16888293.

25. Besedovsky L, Lange T, Born J. Sleep and immune function. Pflugers Arch. 2012; 463(1): 121-137, doi: 10.1007/s00424-011-1044-0, indexed in Pubmed: 22071480.

26. Kim W, Lee J, Ha J, et al. Association between sleep duration and subclinical thyroid dysfunction based on nationally representative data. J Clin Med. 2019; 8(11), doi: 10.3390/jcm8112010, indexed in Pubmed: 31752113.

27. Hughes RJ, Sack RL, Lewy AJ. The role of melatonin and circadian phase in age-related sleep-maintenance insomnia: assessment in a clinical trial of melatonin replacement. Sleep. 1998; 21(1): 52-68, doi: 10.1093/sleep/21.1.52.

28. Tsatsoulis A. The role of stress in the clinical expression of thyroid autoimmunity. Ann N Y Acad Sci. 2006; 1088: 382-395, doi: 10.1196/annals.1366.015, indexed in Pubmed: 17192582.
29. Innatowicz $P$, Drywień $M$, Wątor $P$, et al. The importance of nutritional factors and dietary management of Hashimoto's thyroiditis. Ann Agric Environ Med. 2020; 27(2): 184-193, doi: 10.26444/ aaem/112331, indexed in Pubmed: 32588591.

30. Mansournia N, Mansournia MA, Saeedi S, et al. The association between serum 250HD levels and hypothyroid Hashimoto's thyroiditis. J Endocrinol Invest. 2014; 37(5): 473-476, doi: 10.1007/ s40618-014-0064-y, indexed in Pubmed: 24639121.

31. Asik M, Gunes F, Binnetoglu E, et al. Decrease in TSH levels after lactose restriction in Hashimoto's thyroiditis patients with lactose intolerance. Endocrine. 2014; 46(2): 279-284, doi: 10.1007/ s12020-013-0065-1, indexed in Pubmed: 24078411.

32. Ruchała M, Szczepanek-Parulska E, Zybek A. The influence of lactose intolerance and other gastro-intestinal tract disorders on L-thyroxine absorption. Endokrynol Pol. 2012; 63(4): 318-323, indexed in Pubmed: 22933169.

33. Krysiak R, Szkróbka W, Okopień B. The Effect of Gluten-Free Diet on Thyroid Autoimmunity in Drug-Na ve Women with Hashimoto's Thyroiditis: A Pilot Study. Exp Clin Endocrinol Diabetes. 2019; 127(7): 417-422, doi: 10.1055/a-0653-7108, indexed in Pubmed: 30060266.

34. Szostak-Węgierek D, Bednarczuk T. The validity of gluten- free diet in Hashimoto's thyroiditis: statement of the Expert Committee of the Section of Medical Dietetics of the Polish Society for Parenteral, Enteral Nutrition and Metabolism (POLSPEN). Postępy Żywienia Klinicznego. 2018; 2(47): 33-47.

35. Pastusiak K, Michałowska J, Bogdański P. Nutrition treatment in thyroid diseases. Forum Zaburzeń Metabolicznych. 2017; 8(4): 155-160.

36. Degirmenci PB, Kirmaz C, Oz D, et al. Allergic rhinitis and its relationship with autoimmune thyroid diseases. Am J Rhinol Allergy. 2015; 29(4): 257-261, doi: 10.2500/ajra.2015.29.4189, indexed in Pubmed: 26067918.

37. Amino N, Hidaka Y, Takano T, et al. Association of seasonal allergic rhinitis is high in Graves' disease and low in painless thyroiditis. Thyroid. 2003; 13(8): 811-814, doi: 10.1089/105072503768499699, indexed in Pubmed: 14558923.

38. Takeoka K, Hidaka Y, Hanada $\mathrm{H}$, et al. Increase in serum levels of autoantibodies after attack of seasonal allergic rhinitis in patients with Graves' disease. Int Arch Allergy Immunol. 2003; 132(3): 268-276, doi: 10.1159/000074309, indexed in Pubmed: 14646389.

39. Dong BJ. How medications affect thyroid function. West J Med. 2000; 172(2): 102-106, doi: 10.1136/ewjm.172.2.102, indexed in Pubmed: 10693372.

40. Sugumar R, Kannan S, Asirvatham AR, et al. Absence of iodine/ /iodide in cough/expectorant medications: a true disclaimer or not? Indian J Nucl Med. 2018; 33(1): 84-85, doi: 10.4103/ijnm. IJNM_105_17, indexed in Pubmed: 29430128.

41. Backer $\mathrm{H}$, Hollowell J. Use of iodine for water disinfection: iodine toxicity and maximum recommended dose. Environ Health Perspect. 2000; 108(8): 679-684, doi: 10.1289/ehp.00108679, indexed in Pubmed: 10964787.

42. Freedman DO, Chen LH. Vaccines for International Travel. Mayo Clin Proc. 2019; 94(11): 2314-2339, doi: 10.1016/j.mayocp.2019.02.025, indexed in Pubmed: 31685156.

43. Heng S, Hughes B, Hibbert M, et al. Traveling with cancer: a guide for oncologists in the modern world. J Glob Oncol. 2019; 5: 1-10, doi: 10.1200/JG0.19.00029, indexed in Pubmed: 31291137.

44. Pokhrel B, Aiman W, Bhusal K. Thyroid Storm. In: StatPearls. Treasure Island (FL): StatPearls Publishing 2021. 